EPJ Web of Conferences 59, 17001 (2013)

DOI: $10.1051 /$ epjconf/20135917001

(C) Owned by the authors, published by EDP Sciences, 2013

\title{
Channeling of relativistic laser pulses in underdense plasmas and subsequent electron acceleration
}

\author{
N. Naseri ${ }^{1}$, D. Pesme ${ }^{1,2, a}$, W. Rozmus ${ }^{1}$, V.Yu. Bychenkov ${ }^{3}$ and K. Popov ${ }^{4}$ \\ 1 Theoretical Physics Institute, University of Alberta, Edmonton T6G 2J1, Alberta, Canada \\ 2 Centre de Physique Théorique, CNRS, Ecole Polytechnique, 91128 Palaiseau Cedex, \\ France \\ 3 P. N. Lebedev Physics Institute, Russian Academy of Science, Moscow 119991, Russia \\ 4 Department of Physics, University of Ottawa, Ottawa, Ontario, Canada
}

\begin{abstract}
This contribution is concerned with the nonlinear behavior of a relativistic laser pulse focused in an underdense plasma and with the subsequent generation of fast electrons. Specifically, we study the interaction of laser pulses having their intensity $\mathrm{I} \lambda^{2}$ in the range $\left[10^{19}, 10^{20}\right] \mathrm{W} / \mathrm{cm}^{2} \mu \mathrm{m}^{2}$, focused in a plasma of electron density $\mathrm{n}_{0}$ such that the ratio $\mathrm{n}_{0} / \mathrm{n}_{\mathrm{c}}$ lies in the interval $\left[10^{-3}, 2 \times 10^{-2}\right], \mathrm{n}_{\mathrm{c}}$ denoting the critical density; the laser pulse power $\mathrm{P}_{\mathrm{L}}$ exceeds the critical power for laser channeling $\mathrm{P}_{\mathrm{ch}}$. The laserplasma interaction in such conditions is investigated by means of 3D Particle in Cell (PIC) simulations. It is observed that the laser front gives rise to the excitation of a surface wave which propagates along the sharp boundaries of the electron free channel created by the laser pulse. The mechanism responsible for the generation of the fast electrons observed in the PIC simulations is then analyzed by means of a test particles code. It is thus found that the fast electrons are generated by the combination of the betatron process and of the acceleration by the surface wave. The maximum electron energy observed in the simulations with $\mathrm{I} \lambda^{2}=10^{20} \mathrm{~W} / \mathrm{cm}^{2} \mu \mathrm{m}^{2}$ and $\mathrm{n}_{0} / \mathrm{n}_{\mathrm{c}}=2 \times 10^{-2}$ is $350 \mathrm{MeV}$.
\end{abstract}

The interaction of high-energy laser pulses at relativistic intensities with underdense plasmas is an important area of research, for the studies of the fundamental aspects of the relativistic laser-plasma interaction physics, for its physical applications [1] in particle acceleration and in radiation sources, and for the fast ignition scenario [2] of the inertial confinement fusion. Several experiments, including some very recent ones $[3,4]$ on the propagation of sub-picosecond and picosecond pulses in gas jet plasmas, have motivated the examination of basic nonlinear physical processes such as relativistic selffocusing [5], self-modulation and induced focusing [6], laser pulse channeling [7] and channel stability [8], surface wakes [9] and the electron acceleration in the evacuated channels [10]. We have studied all these processes by means of 3D PIC simulations and analytical theory.

We made extensive 3D simulations for an incident laser beam characterized by the initial FWHM $\mathrm{w}_{0}=10 \mu \mathrm{m}$, laser intensity $\mathrm{I}=5 \times 10^{19} \mathrm{~W} / \mathrm{cm}^{2}$, focused in a plasma of density $\mathrm{n}_{0}=10^{-3} \mathrm{n}_{\mathrm{c}}$ along the $\mathrm{z}$-direction; the laser field was linearly polarized along the $\mathrm{x}$-direction, its wavelength $\lambda_{0}$ was $\lambda_{0}=1 \mu \mathrm{m}$, and $\mathrm{n}_{\mathrm{c}}$ denotes the corresponding critical density. With these parameters, the laser beam power is $28 \mathrm{TW}$, and the ratio $\mathrm{P}_{\mathrm{L}} / \mathrm{P}_{c h}$ is $\mathrm{P}_{\mathrm{L}} / \mathrm{P}_{c h}=1.6 ; \mathrm{P}_{\mathrm{L}}$ and $\mathrm{P}_{c h}=1.09 \mathrm{P}_{\mathrm{c}}$ denote the incident laser wave power and the power threshold for channeling, respectively, $\mathrm{P}_{\mathrm{c}}$ being the usual critical power for self-focusing. Figure 1 presents the numerical results obtained with the following parameters: laser pulse duration $\tau_{\mathrm{L}}=400 \mathrm{fs}$, physical time $\tau_{\text {sim }}$ corresponding to the PIC simulations $\tau_{\text {sim }}=2200 \mathrm{fs}$.

In this regime, the full electron evacuation takes place very rapidly, leading to the formation of a channel void of electrons, as predicted by Kim et al. [11]. The channel radius $r_{c h}$ is $r_{c h}=12 \mu \mathrm{m}$,

\footnotetext{
ae-mail: pesme@cpht.polytechnique.fr
}

This is an Open Access article distributed under the terms of the Creative Commons Attribution License 2.0, which permits unrestricted use, distribution, and reproduction in any medium, provided the original work is properly cited. 


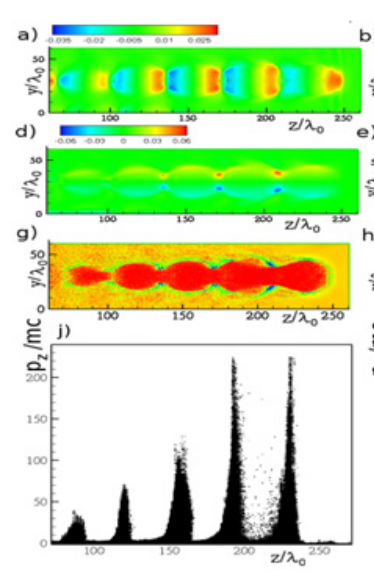

$861 \mathrm{fs}$

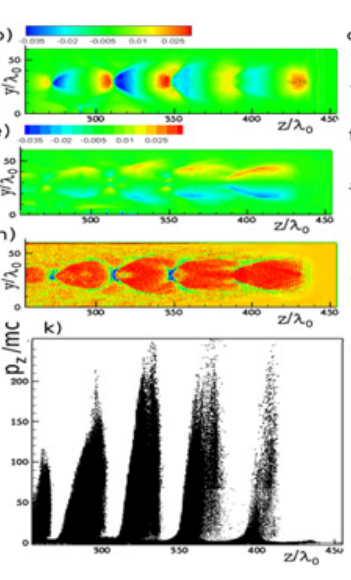

1507 fs

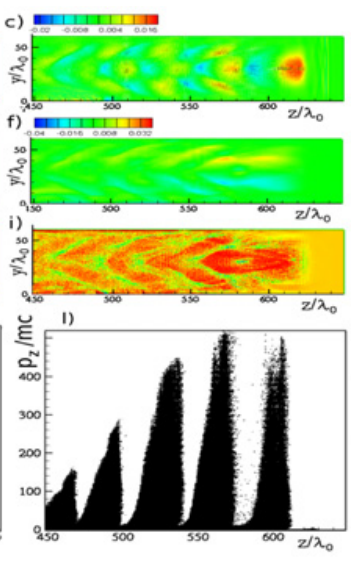

$2154 \mathrm{fs}$

Figure 1. Results from the PIC simulations: contour plots of $E_{z}(a, b, c), E_{y}(d, e, f)$, and electron density (g, h, i) in the y-z plane at three different times: $861 \mathrm{fs}$ (left column), $1507 \mathrm{fs}$ (middle column) and $2154 \mathrm{fs}$ (right column). In the lowest boxes are shown the longitudinal momentum $\mathrm{p}_{\mathrm{z}} / \mathrm{mc}$ as a function of the longitudinal coordinate $\mathrm{z}$.

in very close agreement with the prediction $r_{e q}=11.6 \mu \mathrm{m}$ of Ref. [11]. The electron density profile varies very rapidly in the vicinity of the cylinder located at $\mathrm{r}=\mathrm{r}_{\mathrm{ch}}$ : it is vanishing within the channel $\mathrm{r}<\mathrm{r}_{\mathrm{ch}}$, raises at its maximum at $\mathrm{r}=\mathrm{r}_{\mathrm{ch}}$, and quickly decays to the background value for $\mathrm{r}>r_{\mathrm{ch}}$. Such a steep density profile at the channel wall is favorable for the excitation of surface waves (SW) $[11,12]$. A rapid growth of a SW is indeed observed in our PIC simulation, as it can be seen in Figure 1 which shows in the yz-plane the contour plots of the z-component, $\mathrm{E}_{\mathrm{z}}$, of the electromagnetic field [Figs. 1(a, b, c)], the contour plots of its transverse y-component, $E_{y}[$ Figs. 1(d, e, f)], and the contour plots of the electron density [Figs. $1(\mathrm{~g}, \mathrm{~h}, \mathrm{i})$ ], at the times $\mathrm{t}=861 \mathrm{fs}, 1507 \mathrm{fs}$, and $2154 \mathrm{fs}$ (the laser electric field, being linearly polarized along the direction $\mathrm{x}$, has no component in the plane $\mathrm{xz}$ shown in this figure). In Fig. 1(d, e, f), it can be clearly seen that, at a given y, the amplitude of $\mathrm{E}_{\mathrm{y}}$ oscillates as a function of $\mathrm{z}$ with the characteristic wavelength $\lambda_{\mathrm{SW}} \approx 36 \mu \mathrm{m}$ around a nonzero averaged value. These oscillations show that the electric field $\mathrm{E}_{\mathrm{y}}$ is the sum of the $\mathrm{y}$-component of the SW oscillating electric field $\mathbf{E}_{\mathrm{SW}}$ and of the positive charge separation electric field $\mathbf{E}_{\mathrm{CS}}$ in the electronfree channel.

It is remarkable that a single mode, characterized by the wave number $\mathrm{k}_{\mathrm{SW}}=2 \pi / \lambda_{\mathrm{SW}}$, grows with time, whereas the SW dispersion relation predicts a continuum of wave-numbers for the SW perturbations. We checked that the mechanism selecting the wave number $\mathrm{k}_{\mathrm{SW}}$ of the SW excited by the laser wave front is the process described in Ref. [13]; namely, $\mathrm{k}_{\mathrm{SW}}$ is defined by the implicit equation, $\omega_{\mathrm{SW}}-\mathrm{k}_{\mathrm{SW}} \mathrm{V}_{\mathrm{GL}}=\omega_{0}-\mathrm{k}_{0} \mathrm{~V}_{\mathrm{GL}}$, where $\omega_{\mathrm{SW}}$ denotes a solution to the $\mathrm{SW}$ dispersion relation for the wave-number $\mathrm{k}_{\mathrm{SW}} ; \mathrm{V}_{\mathrm{GL}}$ denotes the laser wave group velocity. Using a linear description for the propagating front of the laser pulse, the latter equation leads to the solution $\lambda_{\mathrm{Sw}} / \lambda_{0}=36$, in excellent agreement with our simulation results. Within the same linear approximation for the laser pulse front, one may compute the $\mathrm{SW}$ phase velocity, $\mathrm{V}_{\varphi \mathrm{SW}}$. One obtains $V_{\varphi \mathrm{SW}} / \mathrm{c}=\mathrm{V}_{\mathrm{GL}} / \mathrm{c}-\left(\lambda_{\mathrm{SW}} / \lambda_{0}\right)$ $\left(\mathrm{V}_{\varphi \mathrm{L}} / \mathrm{c}\right)\left(\mathrm{n}_{0} / \mathrm{n}_{\mathrm{c}}\right), \mathrm{V}_{\varphi \mathrm{L}}$ denoting the laser wave phase velocity; for our parameters, this relation leads to $\mathrm{V}_{\varphi \mathrm{SW}} / \mathrm{c}=0.96$, corresponding to the relativistic Lorentz factor $\gamma_{\varphi \mathrm{SW}} \approx 3$, in very close agreement with our numerical results $\mathrm{V}_{\varphi \mathrm{SW}} / \mathrm{c}=0.95$ and $\gamma_{\varphi \mathrm{SW}} \approx 3$. Thus the PIC results are all close to the theoretical predictions [13] concerning the mechanism selecting the SW wavelength.

In the lowest boxes of Figure 1 are displayed the normalized longitudinal momentum $\gamma_{\mathrm{z}} \equiv \mathrm{p}_{\mathrm{z}} / \mathrm{mc}$ as a function of the longitudinal coordinate $\mathrm{z}$. Two characteristic features can be stressed from these figures: (i) the accelerated electrons form periodic bunches whose periodicity length is the SW wavelength 


\section{IFSA 2011}

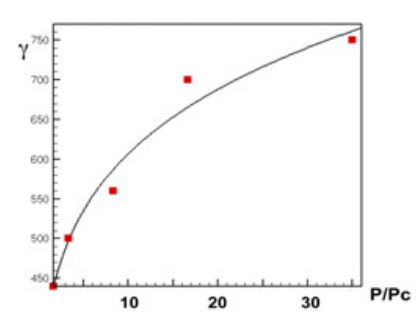

Figure 2. The maximum electron Lorentz factor $\gamma$ obtained in the test-particle calculations carried out with the parameters $\lambda_{0}=1 \mu \mathrm{m}$, initial FWHM $\mathrm{w}_{0}=7 \mu \mathrm{m}$, as a function of the parameter $\mathrm{P} / \mathrm{P}_{\mathrm{c}}$.

$\lambda_{\mathrm{SW}}$; (ii) at the time $\mathrm{t}=1507 \mathrm{fs}$, the maximum electron $\gamma_{\mathrm{z}}$-factor is in the order of $\gamma_{\mathrm{z}} \approx 250$. When plotting the corresponding electron distribution function, one observes a characteristic tail of fast electrons in the range $[30,250]$ for $\gamma_{z}$. The question is therefore to determine the physical mechanism responsible for this relativistic tail. In view of the periodic structure of $\gamma_{z}$ as a function of the longitudinal coordinate $\mathrm{z}$, it is natural to begin with putting estimates on the maximum energy that the electrons can gain when the acceleration mechanism is simply the linear wave-particle interaction with the surface wave along the parallel direction $\mathrm{z}$. Following the Reference [14], the maximum Lorentz factor $\gamma_{\mathrm{zmaxS}}$ that an electron may acquire because of trapping in a periodic electrostatic wave in the presence of a laser wave, is given by $\left(\gamma_{\mathrm{zmaxSW}}\right)^{2}=\left(\gamma_{\mathrm{L}}\right)^{2}+\left(\mathrm{p}_{\mathrm{zmaxSW}} / \mathrm{mc}\right)^{2}$, where the term $\left(\gamma_{\mathrm{L}}\right)^{2}$ accounts for the motion caused by the laser wave, $\left(\gamma_{\mathrm{L}}\right)^{2} \equiv 1+\left(\mathrm{a}_{0}\right)^{2} / 2$, and $\mathrm{p}_{\mathrm{zmaxSw}} / \mathrm{mc}$ is the maximum normalized electron momentum in the surface wave alone; $\mathrm{a}_{0} \equiv \mathrm{eE}_{\mathrm{L}} /\left(\mathrm{m} \omega_{0} \mathrm{c}\right)$ denotes the usual normalized laser electric field amplitude $\mathrm{E}_{\mathrm{L}} \cdot \mathrm{p}_{\mathrm{zmaxSw}} / \mathrm{mc}$ is given by $\mathrm{p}_{\mathrm{zmaxSW}}=\beta_{\phi} \gamma_{\phi}\left(\gamma_{\mathrm{L}}+\gamma_{\phi} \Delta \Phi_{\mathrm{swz}}\right)+\gamma_{\mathrm{f}}\left[\left(\gamma_{\mathrm{L}}+\gamma_{\phi} \Delta \Phi_{\mathrm{swz}}\right)^{2}-\right.$ $\left.\left(\gamma_{\mathrm{L}}\right)^{2}\right]^{1 / 2}$, with $\beta_{\phi}=\mathrm{v}_{\phi \mathrm{SW}} / \mathrm{c}$ and $\gamma_{\phi}=1 /\left[1-\left(\beta_{\phi}\right)^{2}\right]^{1 / 2}, \mathrm{v}_{\phi \mathrm{SW}}$ being the SW phase velocity; $\Phi_{\mathrm{swz}}$ denotes the potential from which the SW electric field is derived, $\mathrm{E}_{\mathrm{swz}}=-\partial_{z} \Phi_{\mathrm{swz}}$, and $\Delta \Phi_{\mathrm{swz}}=$ $\Phi_{\text {swzmax }}-\Phi_{\text {swzmin }} \approx 2 \mathrm{E}_{\mathrm{swz}} / \mathrm{k}_{\mathrm{sw}}$. One obtains $\mathrm{p}_{\mathrm{zmaxSw}} / \mathrm{mc} \approx 46$ in the case of an electric field amplitude as large as e $\mathrm{E}_{\mathrm{swz}} /\left(\mathrm{m} \omega_{0} \mathrm{c}\right)=0.03$ leading to $\gamma_{\mathrm{zmaxSw}} \approx 47$, which is well below the $\gamma$-factors $\approx 200$ observed in the PIC simulations. Thus, we may already conclude that the origin of the fast electrons cannot simply be the acceleration caused by the SW electric field.

In order to find out the mechanism responsible for the observed fast electrons, we performed test particles calculations by solving the equations of motion $\mathrm{d} / \mathrm{dt}=-(\mathrm{e} / \mathrm{m})[\mathbf{E}+\mathbf{v} \mathbf{x} \mathbf{B}]$ for prescribed electric and magnetic fields, including the laser electric field $\mathbf{E}_{\mathrm{L}}$, the charge separation field $\mathbf{E}_{\mathrm{CS}}$ of the evacuated ion channels, and the SW electric field $\mathbf{E}_{\mathrm{SW}}$. The longitudinal component of the laser field $\mathrm{E}_{\mathrm{Lz}}$ was given by the divergence-free condition and the magnetic fields $\mathbf{B}$ were calculated from the Maxwell equations. We first checked that all the features observed in the PIC simulations are correctly reproduced, namely the periodic bunching of the fast electrons with $\gamma$-factors as large as 200 , when all the fields are included into the equations of motion. We then verified that the SW alone is not able to accelerate electrons to energies as high as $\gamma_{z}=200$ : indeed, when only keeping the SW electric field, we obtained electron energies no larger than $\gamma_{z}=50$, consistently with the trapped electron dynamics discussed above. We also considered the standard betatron acceleration [10, 15]. This mechanism corresponds to retaining only the laser fields and the transverse charge separation field $\mathbf{E}_{\mathrm{CS}}$ in the equations of motion. The test electrons were introduced initially with the energy $\gamma_{\mathrm{z}} \approx 50$ and the final maximum electron energy corresponded to $\gamma_{z}=140$, i.e. still significantly smaller than $\gamma_{z} \approx 200$ observed in the PIC simulations. Thus the standard betatron effect by itself cannot explain our PIC results. On the other hand, when we combined the betatron process with the acceleration by the longitudinal SW electric field $\mathrm{E}_{\mathrm{swz}}$, we obtained periodic bunches of fast electrons with $\gamma_{\mathrm{z}}$-factors as large as $\gamma_{\mathrm{z}}=200$, independently of the test particles initial energy. Thus, we conclude that the origin of the fast electrons observed in our PIC simulations is the combination of the betatron acceleration with the parallel acceleration due to the longitudinal SW electric field. 


\section{EPJ Web of Conferences}

Finally we did several test particle calculations corresponding to the parameters $\lambda_{0}=1 \mu \mathrm{m}$, initial FWHM $\mathrm{w}_{0}=7 \mu \mathrm{m}$; the intensity $\mathrm{I}$ and the plasma density $\mathrm{n}_{0} / \mathrm{n}_{c}$ were varied in the ranges $[0.62,10] \times 10^{19} \mathrm{~W} / \mathrm{cm}^{2}$ and $\left[10^{-3}, 2 \times 10^{-2}\right]$, respectively, in a way such that $\mathrm{P} / \mathrm{P}_{\mathrm{c}}$ varied in the interval [2.1, 34]; the pulse duration was $\tau_{\mathrm{L}}=600 \mathrm{fs}$. These parameters can be considered to be representative of those of the experiments [3] carried out on the Omega EP facility, namely $\mathrm{n}_{0} / \mathrm{n}_{\mathrm{c}}=0.05$ and $\mathrm{I}=12.8 \times 10^{19} \mathrm{~W} / \mathrm{cm}^{2}$. The results corresponding to our test particle calculations are displayed in Figure 2, showing the maximum Lorentz factor $\gamma$ obtained as a function of $\mathrm{P} / \mathrm{P}_{\mathrm{c}}$, thus demonstrating the efficiency of the mechanism exhibited in this article.

\section{References}

[1] E. Esarey et al., Rev. Mod. Phys. 81, 1229 (2009)

[2] M. Tabak et al., Phys. Plasmas 1, 1626 (1994)

[3] L. Willingale et al., Phys. Rev. Lett. 106, 105002 (2011).

[4] P.M. Nilson et al., New J. Phys. 12, 045014 (2010) ; S.P.D. Mangles et al., Phys. Rev. Lett. 94, 245001 (2005); Z. Najmudin et al., Phys. Plasmas 10, 438 (2003); M. Borghesi et al., Phys. Rev. Lett. 78, 879 (1997)

[5] A.G. Litvak, Sov. Phys. JETP 30, 344 (1969)

[6] T.M. Antonsen, Jr, and P. Mora, Phys. Rev. Lett. 69, 2204 (1992); E. Esarey et al., Phys. Rev. Lett. 72, 2887 (1994); S. V. Bulanov et al., Phys. Rev. Lett. 74, 00319007 (1995); N.E. Andreev et al., Phys. Plasmas 2, 2573, (1995)

[7] Z. Sun et al., Phys. Fluids 30, 526 (1987)

[8] A.B. Borisov et al., Plasma Phys. Control. Fusion 37, 569 (1995) ; V.I. Berezhiani et al., Phys. Rev. E 65, 046415 (2002)

[9] C. Chiou et al., Phys. Plasmas 2, 310, (1995)

[10] A. Pukhov et al., Phys. Plasmas 6, 2847 (1999)

[11] A. Kim et al., Phys. Rev. E 65, 036416, (2002)

[12] N. Naseri et al., Phys. Plasmas 17, 033107, (2010)

[13] N. M. Naumova et al., Phys. Plasmas 8, 4149 (2001)

[14] E. Esarey and M. Piloff, Phys. Plasmas 2, 1435 (1995); C.B. Schroeder et al., Phys. Plasmas 13, 033103 (2006)

[15] D. Tsakiris et al., Phys. Plasmas 7, 3017 (2000) 